\title{
¿ES FACTIBLE HABLAR DE REINGENIERÍA EN LA ADMINISTRACIÓN PÚBLICA?
}

\author{
ROLANDO BOLAÑOS GARITA \\ Escuela de Ciencias de la Administración \\ Universidad Estatal a Distancia, Costa Rica \\ rbolanos@uned.ac.cr
}

\section{RESUMEN}

Recientemente, las ciencias administrativas se han fortalecido mediante una gama de tendencias que representan valiosos instrumentos para la mejor gestión o modernización de las instituciones públicas, como en el caso de la reingeniería. No obstante, no todos estos instrumentos son aplicables para la realidad jurídico-administrativa costarricense, por lo que, previo a su implementación, deben necesariamente adaptarse a la realidad del país para evitar extrapolar tendencias que no generen valor agregado para la administración pública y que carezcan de un sólido fundamento técnico-científico.

PALABRAS CLAVE: ADMINISTRACIÓN PÚBLICA, REINGENIERÍA, GESTIÓN POR PROCESOS.

\section{ABSTRACT}

Recently, Administrative Science have been reinforced by a range of trends that are becoming valuable tools for better management and modernization of public institutions; we are talking about Reengineering. However, not all of these instruments are applicable for the Costa Rican legal-administrative reality, so, before even thinking about its implementation, necessarily, there must be an effort to adapt it to this reality, avoiding to extrapolate trends that do not generate added value to Public Administration and without a solid technical and scientific foundation.

KEYWORDS: PUBLIC ADMINISTRATION, REEINGENIERING, PROCESS MANAGEMENT.

\section{INTRODUCCIÓN}

El presente análisis se consideró oportuno ante la confusión ${ }^{1}$ existente en cuanto al verdadero uso, importancia y radio de acción que ostenta la reingeniería dentro de la gestión administrativa, tanto pública como privada; no obstante el enfoque se centra en la primera gestión referida.

En muchas ocasiones se escucha la expresión: "Hagamos una reingeniería"y de inmediato se vincula con una mejora organizacional; seguramente se ignora su operacionalización, no obstante se infiere que es algo así como un sello de calidad. También se ha homologado con el término re-

1. Incluso la Procuraduría General de la República utiliza el término de manera inadecuada, según el criterio analítico e investigativo del autor; verbigracia, en el Dictamen C-241-2006 se indica lo siguiente:

Conviene aclarar que, según hemos admitido en casos similares (Dictamen C-178-2006 de 9 de mayo de 2006), en materia de empleo público, los procesos integrales de reorganización o reestructuración administrativa, por la transformación de plazas, pueden producir reasignaciones de puestos, tanto en ascenso, como en descenso, pero que al darse en aquel contexto de reingeniería organizativa, es claro que caerían en el ámbito del ejercicio de la potestad de autoorganización, comprendida dentro de la materia administrativa, propia del Consejo Directivo, y no del Decano. La anterior aclaración es conveniente en este caso para no propiciar una invasión o un desapoderamiento de la potestad de autoorganización atribuida a aquél órgano colegiado (sección II.- Sobre lo consultado, párr. 19). 
organización ${ }^{2}$ y por ende tiende a relacionarse con despido de personal o cambios ineludibles en las estructuras administrativas; pero todo lo anterior se encuentra muy alejado de la realidad.

Otro cimiento del análisis surge del expediente legislativo 18.201 Informe: reconstitución del pacto social costarricense con la seguridad social, generado por diputados de diferentes partidos políticos, en razón de las condiciones administrativas y financieras que actualmente presenta la Caja Costarricense del Seguro Social (CCSS). En varias partes del documento se presenta la concreción de una reingeniería como aparente mecanismo catalizador de recursos institucionales, tal y como se desprende del punto 3 de las conclusiones generales: "La crisis de la CCSS es de carácter estructural y de gestión. Esto se refleja en los aspectos financieros, infraestructurales, de servicio, entre otros. La única solución es un proceso profundo de reingeniería institucional" (2012, p.189).

\section{El contexto de la reingeniería}

La reingeniería es una corriente de mejora gerencial que nace a la luz de la publicación Reingeniería de Hammer y Champy (1995); se basa en la necesidad de concretar una transformación profunda y evidente de la gestión administrativa a través de la reorientación de los procesos. Procesos es un término añejo que emana de la Teoría General de Sistemas y que conlleva a valorar la existencia de una serie de pasos o actividades interrelacionadas que generan un valor agregado para con un bien o servicio producido.

Emplea como eje central el autodiagnóstico que debe concretar la organización sobre las activi-

2. En Bolaños (2010), se aclaran una serie de consideraciones de diversa índole que permiten sustentar la valía del término reorganización administrativa, en contraposición con otra terminología carente de fundamento teórico-conceptual. dades que hasta el momento ha desarrollado, cuestionando ¿por qué se está haciendo esto?, ya que si no se cuenta con explicación o fundamento para el desarrollo de una actividad específica, la misma no está generando ningún valor para con el bien o servicio brindado, conforme el interés público que reviste a las instituciones pertenecientes a la administración pública.

Hammer y Champy conciben la reingeniería como: ".. la revisión fundamental y el rediseño radical de procesos para alcanzar mejoras espectaculares en medidas críticas y contemporáneas de rendimiento, tales como costos, calidad, servicio y rapidez" (1995, p.34).

Una reingeniería, según la teoría propuesta, significa empezar de cero, no pegar parches o remiendos en las funciones administrativas desarrolladas; proclama el estructurar una nueva secuencia de acciones administrativas que sí agreguen valor a los bienes o servicios producidos. Este argumento puede ser extremista y desechar toda una serie de acciones que bien podrían no estar enmarañadas en su totalidad y que más bien solo requieren de un ajuste para así lograr su objetivo.

La tendencia, de igual forma, ha tendido a confundirse con un cambio directo de la estructura organizacional, lo cual es un error inducido por la confusión en el uso del término (en otras palabras, consultores que no dominan dicha tendencia y aún así ofrecen sus servicios).

No obstante, al proceder a analizar los mecanismos de gestión por procesos, resulta muy seguro que deba replantearse el organigrama institucional, ya que al reorganizarse las funciones de las diferentes unidades administrativas hacia un enfoque más sistémico y secuencial, podrían trasladarse algunas de ellas que anteriormente se desarrollaban de manera equívoca. De hecho, en la reingeniería se busca más bien que se achate la estructura como mecanismo para 
una mejor comunicación, seguimiento y coordinación de la información y de los procesos en sí.

Esta tendencia tampoco se deslinda del modelo burocrático planteado por el economista y sociólogo alemán Max Weber, tal y como lo externan sus iniciadores:

Las compañías que muy seriamente se empeñan en acabar con las burocracias están tomando el rábano por las hojas. La burocracia no es el problema. Por el contrario, la burocracia ha sido la solución durante los últimos doscientos años. Si a usted no le gusta la burocracia en su compañía, trate de arreglárselas sin ella. El resultado será un caos. La burocracia es el pegamento que mantiene unida la corporación. (Hammer y Champy, 1995, p. 51)

En Hammer y Champy (1995), se reconocen varias figuras que se adaptan a la realidad de una institución pública. Así, cualquier institución pública lleva a cabo procesos, sean de manufactura o prestación de servicios tangibles (conexión de un medidor de agua o luz) o intangibles (promoción social para la conformación de una asociación de desarrollo), por lo que si dentro de la evolución administrativa que está sufriendo el entorno internacional se presiona cada vez más hacia la valoración (y posible incorporación) de este tipo de tendencias en la gestión administrativa pública, deberán coincidir con el contexto político, administrativo y jurídico.

Los actores involucrados serán según Hammer y Champy (1995) los siguientes:

1. Líder de la reingeniería: en el caso de las instituciones públicas sería el jerarca quien debe creer su consecución y brindar no solo su consentimiento, sino también su compromiso para enrumbar a la organización hacia una mejora en sus procesos.
2. Dueño del proceso: jefatura de una unidad administrativa donde se lleva cabo el proceso, o bien coadyuva en parte en su realización.

3. Equipo de reingeniería: grupo de funcionarios dedicados a analizar el proceso y concretar la propuesta para su reingeniería y posterior implementación.

4. Comité directivo: grupo de jefaturas que conforman el nivel gerencial de la organización, cooperando con el jerarca en la propuesta y desarrollo de estrategias para la mejora institucional y, en este caso, para la mejor operacionalización de la reingeniería.

5. Zar de la reingeniería: funcionario responsable de desarrollar técnicas e instrumentos de reingeniería (cuestionarios, manuales, entre otros).

Cada uno de estos actores cumple funciones muy específicas que deben interrelacionarse de manera ordenada y constante para la consecución del cambio. Ahora bien, el éxito de planteamiento propuesto no deviene en que a lo interno de cada institución se nombre a determinado funcionario como líder o zar de la reingeniería, por el contrario, lo que corresponde es reconocer el papel que según la teoría juega el funcionario o funcionarios que participarán en la ejecución de la tendencia.

Según Hammer y Champy (1995), por más grande que sea una institución los procesos medulares que se llevan cabo no son muchos, e indican que son pocas las que realizan más de diez procesos principales, por lo que todas las demás acciones o funciones administrativas son derivadas de ellos y deben concebirse como apoyo; por tanto en el momento de determinar cuáles procesos son los adecuados para aplicárseles la reingeniería, deben formularse algunas preguntas orientadoras según el grado de incidencia para la organización y tomarse en cuenta el si- 
guiente enfoque (adaptado según lo propuesto por los autores de referencia):

I. Disfunción: ¿cuáles procesos están en mayores dificultades?, es decir, cuáles de ellos necesitan mayor rectificación en tanto presentan un estado crítico de ejecución para la buena marcha institucional.

II. Importancia: ¿cuáles procesos ejercen mayor impacto en los usuarios o beneficiarios?, ya que la razón de ser de toda institución pública es el interés público, por lo que resulta lógico analizar el posible espectro de la reingeniería, tomando en cuenta los procesos que más afectan a los receptores mencionados.

III. Factibilidad: ¿cuáles de los procesos que actualmente se llevan a cabo son más susceptibles de una reingeniería?, se debe ser realista y determinar cuáles de ellos se encuentran en un momento propicio para su mejora integral, ya sea por factores internos o externos que rodean o afectan a la organización.

Note el lector que la Reingeniería no puede ser el eje central del expediente legislativo sujeto de abordaje analítico, ya que la CCSS es una institución de gran envergadura no solo en cuanto a su impacto para con el bienestar de la población costarricense, sino, en razón del tamaño de su estructura funcional.

Así las cosas, proponer en un expediente legislativo que la situación de la CCSS será solventada mediante una Reingeniería, es desconocer los elementos contextuales e internos propios de esa institución; e igualmente, se toma a la ligera la técnica per se, la cual según lo anotado hasta este punto, está recubierta de aspectos de particular naturaleza, los cuales deben tomarse con seriedad previo a siquiera proponerlos a cualquier organización como mecanismo de mejora.

\section{Principales productos de la reingeniería}

Conforme con el planteamiento del modelo burocrático propuesto por Weber (1991), una de sus principales aristas es la estandarización de rutinas y procedimientos, lo que significa que la determinación de los elementos más específicos de las funciones en todos los puestos de la organización se compila y autoriza mediante un instrumento técnico institucional, por lo general denominado: manual de procedimientos. El instrumento citado resulta ser el producto ideal de una reingeniería, ya que su objetivo será normalizar o estandarizar una serie de acciones o tareas que resultan indispensables para la adecuada ejecución de los procesos.

El establecimiento de los manuales de procedimientos se nutre de tres tendencias claramente definibles:

a) La administración científica, por cuanto es a través del trabajo de Frederick Winslow Taylor (1980) que se manifiesta la necesidad de establecer y medir científicamente cada una de las acciones o pasos relacionados con las funciones del trabajador.

b) La Teoría General de Sistemas, ya que los componentes del proceso definido en los manuales sigue la orientación sistémica de insumos (recursos materiales o información) que deben transformarse mediante un proceso, para a su vez generar productos (bienes o servicios) que son requeridos por los ciudadanos/asegurados.

c) La calidad total, por cuanto los manuales son parte del establecimiento de estándares que determinan los requisitos mínimos que deben ostentar los productos (bienes o servicios).

En cuanto al término proceso, éste se refiere a un conjunto de actividades que se desarrollan en una secuencia determinada permitiendo obtener 
unos productos o salidas a partir de unas entradas o materias primas (información, solicitudes de servicio, entre otros.) Los procesos pueden ser fabriles (entran y salen materiales transformados) o de gestión (entra y sale información), generando un valor añadido (Bolaños, 2011a).

Los procedimientos, se refieren al nivel de detalle de las actividades que conforman un proceso y que puede variar dependiendo de su complejidad, de los métodos utilizados, así como del nivel de habilidades y formación necesaria para que el personal logre llevarlas a cabo (Bolaños, 2011a).

Un manual de procedimientos (MdP) es un documento institucional que contiene todos los procedimientos de la organización, instrucciones de trabajo y formularios que se consideran necesarios para la mejor ejecución de las actividades.

Según Mora (2007) los manuales constituyen una norma dentro de la propia organización, ya que poseen los siguientes rasgos:

\section{Carácter normativo}

2. Doble función formativa e informativa

3. Activo intangible de la organización

4. Uso frecuente: ayudan en la solución de problemas organizacionales cotidianos (p.15)

De igual forma, la Contraloría General de la República, en su oficio DI-CR-209 (04663), se refiere a los procedimientos en yuxtaposición con el establecimiento de un sistema de control interno dentro de las instituciones públicas:

Además, partiendo de lo que dispone el artículo $8^{\circ}$ de la Ley General de Control Interno en cuanto a que el sistema de control interno constituye la serie de acciones ejecutadas por la administración activa, diseñadas para proporcionar seguridad en la consecución, entre otros, del objetivo de proteger y conservar el patrimonio público contra cualquier pérdida, despilfarro, uso indebido, irregulari- dad o acto ilegal, y considerando que el artículo 10 de esa misma Ley dispone que es responsabilidad del jerarca y del titular subordinado establecer, mantener, perfeccionar y evaluar el sistema de control interno institucional, y asimismo, que es responsabilidad de la administración activa realizar las acciones necesarias para garantizar su efectivo funcionamiento, ello conduce a concluir que la administración está en el deber y legalmente facultada, para tomar medidas sobre el particular, lo que incluye la emisión, instauración y ejecución de políticas, normas, procedimientos, etc., que particularicen ese deber general en los asuntos específicos y muy propios de su ámbito institucional (2004, p.6).

De manera más directa, la exhortación por parte de la Contraloría para la emisión de los manuales de procedimientos, se basa en lo establecido en la Norma 4.2 Requisitos de la Actividades de control, requisito e) Documentación, de las Normas de Control Interno para el Sector Público N-22009-CO-DFOE, misma que establece:

Documentación. Las actividades de control deben documentarse mediante su incorporación en los manuales de procedimientos, en las descripciones de puestos y procesos, o en documentos de naturaleza similar. Esa documentación debe estar disponible, en forma ordenada conforme con criterios previamente establecidos, para su uso, consulta y evaluación (2009, p. 14).

Se debe considerar la posibilidad de que el Ente Contralor haya cometido la omisión de no promover, fortalecer y fiscalizar la organización de la administración pública mediante una gestión por procesos, ya que así se tendría todo un engranaje o sistema administrativo público encauzado y dirigido hacia una misma dirección; no como se encuentra actualmente, que la mayoría de instituciones cuenta con una estructura funcional que corta las acciones administrativas hasta donde los 
grados de responsabilidad lo permiten, en lugar de contar con acciones continuas capaces de ser observadas, medidas y corregidas con facilidad, dándoles un seguimiento continuo. En otras palabras, es desarrollar mecanismos administrativos sin contemplar qué puede o quiere hacer la institución, sino, en qué, cómo y cuándo se requieren los bienes o servicios.

\section{La aplicabilidad de la técnica en la Caja costarricense de Seguro Social}

Los argumentos señalados indican que la receta propuesta en el expediente legislativo 18.201 debe replantearse de manera inmediata, ya que no se puede pretender aplicar una técnica como la reingeniería sin antes haber participado a los niveles decisores y administrativos de la CCSS, que serán en definitiva los encargados de valorar no solo su puesta en práctica, sino también de evaluar sus resultados en cuanto a la atención de la seguridad social.

Resulta importante aclarar también que a nivel internacional se han aunado esfuerzos para que los gobiernos de Iberoamérica reconozcan la importancia de modernizar su gestión administrativa, dándole un matiz sistémico y no trastocado, pero que sobre todo se vuelque hacia la mejor prestación de los servicios públicos requeridos por los usuarios y/o beneficiarios. Así, la gestión por procesos promulgada en la Carta Iberoamericana de Calidad en la Gestión Pública señala:

45. La gestión por procesos implica el desarrollo de las actuaciones siguientes:

a. La identificación de los requerimientos, necesidades y expectativas, de los diferentes destinatarios de las prestaciones y servicios públicos, así como de otros posibles grupos de interés.

b. La identificación de todos los procesos necesarios para la prestación del servicio público y la adecuada gestión de la organización: procesos clave u operativos, de apoyo a la gestión y estratégicos; lo que se denomina mapa de procesos.

c. La definición del objetivo de cada uno de los procesos, así como de los beneficios que aporta a los grupos de interés a los que va dirigido.

d. La definición secuencial detallada y precisa de las diferentes actividades que componen el proceso o procedimiento concreto, para el cumplimiento de los diferentes requerimientos, y en su caso su diagrama.

e. La definición de las obligaciones, así como de las autoridades y directivos encargados.

f. La definición de indicadores que permitan la medición y control del desarrollo de la marcha adecuada del proceso.

g. La definición y desarrollo de un sistema de gestión que permita el seguimiento, evaluación y mejora continua de la calidad de los procesos, y la prestación del servicio.

h. La implantación de sistemas de gestión normalizados o estandarizados (2008, p.2021. Énfasis del autor).

Hasta este punto valga también rescatar los esfuerzos que el Ministerio de Planificación Nacional y Política Económica (MIDEPLAN) ha vertido mediante ciertos documentos técnico-orientadores para lograr que las instituciones concreten acciones de organización administrativa coherente, sistémica y unitaria en materia de gestión por procesos, buscando la mejora en la gestión y los resultados previstos.

Así las cosas, a la fecha se han emitido los siguientes documentos:

- Guía para el levantamiento de procesos

- Guía para la elaboración de diagramas de flujos 
- Guía para el rediseño de procesos

- Guía de manuales administrativos

Todas y cada una de estas guías han sido construidas, según se desprende de la presentación incluida en ellas, con el afán de apoyar y fortalecer el Sistema Nacional de Planificación a través de las unidades de planificación institucional, mismas que: "deben conducir y acompañar los procesos de modernización institucional, según se desprende del Decreto 33713-MP-PLAN-MTSS" (MIDEPLAN, 2007, p. 4).

Es importante dejar claro que no importa si la normalización de los procesos o acciones administrativas desarrolladas por las instituciones públicas se encuentra recogida en decretos ejecutivos, manuales, circulares u otros. Lo que no debe obviarse es que la normalización sea respetuosa de lo plasmado igualmente en la Ley de Protección al Ciudadano sobre el exceso de requisitos y trámites administrativos 8220 y en el Reglamento sobre los Programas de Mejora Regulatoria y Simplificación de Trámites dentro de la Administración Pública (Decreto Ejecutivo 33678-MP-MEIC). Así, cuando los técnicos o profesionales formulen los instrumentos antes enunciados, deben reconocer la necesidad de coordinación previa con otras posibles instancias receptoras de los bienes o servicios producidos, no solo agilizar trámites para mejorar según principios de eficiencia y eficacia pública, sino cumplir con la normativa establecida con el propósito de no enmarañar la gestión pública, aspecto que riñe totalmente con una adecuada reingeniería.

Merece la pena indicar que en el expediente legislativo 18.201 no se menciona la Ley 8220, como tampoco se nota que se hayan valorado los instrumentos generados por MIDEPLAN para la mejor gestión administrativa. Por lo tanto, el proyecto para la CCSS carece de un abordaje técnico-administrativo holístico, lo permite cuestionar el impacto en la mejora de la insti- tución y en la prestación de atención médicosocial a los asegurados.

Así bien, cuando se activan procesos de reorganización administrativa, se debe tener conciencia de que el fin último es mejorar las condiciones de eficiencia y eficacia organizacional. Señala Meoño (1980) sobre la eficiencia:

El concepto de eficiencia es importante en lo que se refiere al reconocimiento de que las acciones públicas deben y pueden prestarse dentro de límites razonables de productividad institucional e interinstitucional, evitando situaciones innecesarias de "desperdicio de recursos", pero no como medida de éxito de la gestión pública, como sí ocurre en el caso del empresario privado. (pp. 54-55)

En relación con la eficacia, Meoño plantea:

Una institución pública, o mejor un programa público, puede ser efectivo o eficaz porque logra sus objetivos, pero con un nivel de ineficiencia o de "desperdicio" alto: esto puede darse porque usualmente se incurre en una serie de costos duplicativos o innecesarios que, de haber racionalidad en el planeamiento y administración del programa, no existirían (Meoño, 1980, p.55).

Si no se tiene claridad sobre la terminología de referencia, cualquier procedimiento de transformación y modernización administrativa sería ilusorio, infundado, carente de enfoque científico, por ende, inservible, con mayor razón si se trata de la Caja Costarricense de Seguro Social (CCSS) y la correspondiente prestación de servicios médico-sociales a los asegurados.

En relación con el aspecto precitado, resulta preocupante encontrar recomendaciones como las siguientes en el expediente legislativo 18.201: 
1.1 Instar a la Presidencia de la República a fin de que convoque a los sectores representados en la Junta Directiva de la CCSS, para suscribir un acuerdo nacional que implique la restructuración del órgano colegiado, excepto su Presidencia Ejecutiva, con el propósito de realizar un proceso de reingeniería institucional.

1.2 Solicitar la contratación de un (a) consultor externo de alto nivel gerencial y con experiencia en procesos de reingeniería, a efectos de que acompañe a la Presidencia Ejecutiva de la CCSS en el proceso de restructuración e implementación de medidas tendientes a la mejora de la gestión institucional. (2012, p. 200)

2.1 Solicitar a las autoridades superiores de la CCSS una reingeniería efectiva, con el objetivo de resolver el problema estructural-funcional que se ha generado a la luz de que el desequilibrio financiero en el SEM se produce precisamente porque los gastos crecen más rápidamente que los ingresos, especialmente, en el rubro de servicios personales, poner orden para lograr la optimización de dichos recursos. Además, la reingeniería deberá incluir la mejora en la prestación de los servicios al asegurado.

2.2 Nombrar equipo gestor de alto nivel que coadyuve a la Presidencia Ejecutiva y al consultor externo en la ejecución de la reingeniería institucional. (2012, p. 202. Énfasis del autor).

En la cita anterior, nótese el uso indiscriminado de los términos reestructuración y reingeniería, lo cual genera la duda sobre el sentido epistemológico de los mismos, al punto de que se recomienda el segundo de ellos como "única solución" para la situación que enfrenta hoy en día la CCSS.

Como ya se había mencionado, si bien la reingeniería engrosa la lista de tendencias administrativas que permiten mejorar la gestión de las instituciones, sean públicas o privadas, debe adaptarse al contexto institucional donde se lleve a cabo y, a la vez, desecharse la creencia infundada de que generar reingeniería provoca indefectiblemente el cambio en las estructuras organizativas o al despido de personal, aún cuando estos pueden ser efectos derivados de un proceso de esa naturaleza, pero nunca serán su objetivo primordial o implícito.

En este contexto, se ha observado el rechazo que prácticas como la planteada han sufrido por parte de reconocidos académicos costarricenses, que anuncian la imposibilidad de que comulgue con la realidad de las organizaciones públicas. De igual forma, son escépticos de su éxito para lograr una mejor ejecución de los objetivos y funciones en dichas instancias.

No obstante, se considera la posibilidad de que esto se deba a la confusión que se ha mantenido a lo largo del tiempo en cuanto a su implementación, aunado al hecho de que surgen "consultores" que proclaman su experiencia en el tema, la cual han aplicado de manera equivocada principalmente en la empresa privada, hecho que ha generado un desfase en relación con una posible ejecución en ministerios, municipalidades u otro tipo de organizaciones de la administración pública.

Se deben cerrar flancos para la ineficacia, la ineficiencia y la corrupción en las instituciones públicas costarricenses, pero no a costa de desechar herramientas de valor como la reingeniería. No es conveniente aislarse de las tendencias modernas solo por el hecho de haber sido concebidas en otras latitudes, ya que si bien se ha defendido la no extrapolación de "modas administrativas" importadas de países con realidad disímiles a la costarricense, tampoco se debe caer en la miopía de no "tropicalizar" aquellas que sí pueden surtir efecto en pro de la gestión institucional de la "cosa pública", como también es el caso de la normalización y(o) el desarrollo organizacional (Bolaños, 2010). 


\section{CONCLUSIONES}

Existe una tendencia actual en la administración pública costarricense a decantarse por la contratación de consultorías privadas para concretar procedimientos de reorganización administrativa que mejoren el funcionamiento de las instituciones públicas.

La práctica mencionada no necesariamente es la más conveniente, por cuanto no siempre los consultores tienen el conocimiento y la sagacidad para liderar transformaciones de amplio espectro, como también podrían carecer de un conocimiento básico de tendencias administrativas como la reingeniería o el desarrollo organizacional. Tales falencias pueden resultar únicamente en el pago de las consultorías, pero sin lograr contar finalmente con un producto de valor agregado para la instancia contratante.

El marco referencial se ha centrado en Hammer y Champy, por considerar que el resto de trabajos u obras editadas no constituyen más que adaptaciones o interpretaciones que otros autores brindan a la obra original referida. No obstante, Jofré (2005) presenta una revisión sobre la tendencia analizada, la cual agrega un valor per se, pues detalla algunas de las críticas y revisiones que ha sufrido la reingeniería, tal como la señalada por Hammer (2005): "Por mis antecedentes de ingeniero, no presté suficiente atención a la dimensión humana. He aprendido que es un factor vital" (p.237).

A pesar de eso, la reingeniería en su esencia (la presentada en la obra original de Hammer y Champy), resulta ser piedra angular de una técnica mayor como lo es el desarrollo organizacional, la cual es el brazo operativo de una reorganización administrativa que, desde cualquier perspectiva, debe ser ejecutada por las unidades de planificación institucional (Bolaños, 2012, p.472).

Finalmente, se considera que todo aquél que se interese en esta temática porque su desempeño laboral en la administración pública así lo exija, sea en la práctica o en la investigación, deberá inexo- rablemente acceder al documento generado por la Subsecretaría de la Gestión Pública (SGP) de Argentina: Manual para el Análisis, Evaluación y Reingeniería de los Procesos en la Administración Pública (2006), el cual goza de una excelente claridad teórica, conceptual y metodológica para activar una estrategia de reingeniería en cualquier aparato estatal, sobre todo porque desarrolla el tema de las actividades-fin y las actividades-medio. Sobre las primeras especifica:

...refieren a los objetivos que debe cumplir la institución, de acuerdo con la misión formalmente establecida por medio de su norma constitutiva y se corresponde con los productos finales que arroja el sistema. Estos son denominados sistemas sustantivos u operativos y están establecidos con base en la especificidad de los productos que debe obtener mediante su sistema específico de producción (SGP, 2006, p.7).

Precisamente, la cita anterior aclara meridianamente el objetivo primigenio de una reingeniería, es decir, la mejora en los procesos productivos institucionales, que para las instituciones públicas se refieren a la prestación de bienes o servicios para la población meta, la cual debe explicitarse o bien extraerse de sus objetivos o funciones legales o, en última instancia, de la actividad $^{3}$ de cada ente.

Por ende, previo a propulsar el expediente legislativo 18.201, debe abordarse más profusamente lo que significa y comprende una reingeniería en el contexto de la CCSS, y por supuesto de todas sus consideraciones, sobre todo las metodológicas, pues la razón primera y última será una mejor atención al receptor (asegurado, principalmente) en cuanto a los servicios médico-sociales.

3. El término "actividad", específicamente en lo tocante a un ente público, ha sido desarrollado profusamente por Meoño (2008), por lo que referimos al lector su obra, titulada Guía para un excelente gobierno y... para un ciudadano menos indolente, en su Capítulo 3. 
Lo anterior conduce a desechar cualquier abordaje casuístico e improvisado que comprometa recursos públicos, como es el caso de consultorías que poco aportan al valor público de la CCSS.

\section{Referencias}

Área de Modernización del Estado - MIDEPLAN. (2007). Guía para el rediseño de Procesos. San José: Autor.

Área de Modernización del Estado MIDEPLAN. (2009). Guía para la elaboración de Diagramas de Flujo. San José: Autor.

Área de Modernización del Estado MIDEPLAN. (2009). Guía para el levantamiento de Procesos. San José: Autor.

Área de Modernización del Estado MIDEPLAN. (2009). Guía de Manuales Administrativos. San José: Autor.

Asamblea Legislativa. (2012). Expediente Legislativo 18.201: Informe Reconstitución del pacto social costarricense con la seguridad social. Recuperado de: http://www.asamblea. go.cr/Informe\%20de\%20comisiones\%20especiales/Informe\%20de\%20mayoría\%20Exp\%2018201\%20CCSS.pdf

Barrantes, R. (2012). Investigación: un camino al conocimiento: un enfoque cuantitativo y cualitativo. -21 reimp. de la 1. ed. —San José: EUNED.

Bolaños, R. (2010). Un abordaje teórico, jurídico y conceptual de los términos Reorganización y Reestructuración Administrativa en Costa Rica. Revista Nacional de Administración. 1 (1), 149-158.

Bolaños, R. (2011a). Algunas consideraciones sobre Normalización en la Administración Pública. Revista de Ciencias Económicas, 29 (1), 479-491.

Bolaños, R. (2011b). El Desarrollo Organizacional como estrategia para la modernización en la Administración Pública. Revista Nacional de Administración, 2 (1), 135-144.

Bolaños, R. (2012). Evolución administrativa: de las Unidades de Organización y Métodos hasta las Unidades de Planificación Institucional. Revista Centroamericana de Administración Pública. 62-63, 461-477.

Contraloría General de la República (2004). Oficio D/-CR-209 (04663). San José: Autor.

Contraloría General de República (2009). Normas de Control Interno para el Sector Público N-2-2009-CO-DFOE. San José: Autor.

División de Planes y Política Administrativa OFIPLAN. (1978a). Documentos sobre Reforma Administrativa. San José: Autor.
División de Planes y Política Administrativa OFIPLAN. (1978b). Lineamientos para un enfoque integral sobre Planificación Administrativa, Serie Divulgación de Estudios Técnicos No 17. San José: Autor.

Galván, J. (1964). Significado e importancia de la prognosis administrativa. Serie: Ciencia de la Administración No. 226. San José: ESAPAC.

Hammer, M. y Champy, J. (1995). Reingeniería. —6 reimp. de la 1. ed. - Bogotá: Editorial Norma S.A

Hernández, C. (2007). Análisis Administrativo: técnicas y métodos. -5 reimp. de la 1. ed. —San José: EUNED.

Jiménez, W. (1996). Teorías y técnicas organizativas y de representación gráfica de estructuras, de relaciones formales y de procedimientos. San José: EDNASSS.

Jofré, A. (2005). Enfoques gerenciales modernos. -3 reimp. de la 2. ed. - San José: Servicios Profesionales Delphi de Costa Rica.

Meoño, J. (1980). Introducción al análisis de la burocracia pública. San José: EUCR.

Meoño, J. (2008). Guía para un excelente Gobierno... y para un ciudadano menos indolente. San José: IICE-UCR.

Mora, J. J. (2007). Instructivo para la preparación de Manuales de Procedimientos Municipales. -1 reimp. de la 1. ed. San José: CICAP-UCR.

Palacios, A. J. (1984). Introducción al análisis administrativo. San José: EUCR

Subsecretaría de la Gestión Pública. (2006). Manual para el Análisis, Evaluación y Reingeniería de los Procesos en la Administración Pública. Buenos Aires: Autor.

Taylor, F.W. (1980). Principios de la Administración Científica. Buenos Aires: El Ateneo.

Weber, M. (1991). ¿Qué es la burocracia? Buenos Aires: Editorial Leviatán.

Weber, M. (1969). Economía y Sociedad II: Esbozo de sociología comprensiva. -1 reimp. de la 2. ed. México D.F.: Fondo de Cultura Económica.

X Conferencia Iberoamericana de Ministros de Administración Pública y Reforma del Estado (2008). Carta Iberoamericana de Calidad en la Gestión Pública. El Salvador.

Recibido: 22 de enero de 2013 Aceptado: 30 de marzo de 2013 